\title{
Diagnostic Accuracy of Probe-Based Confocal Laser Endomicroscopy and Tissue Sampling by Endoscopic Retrograde Cholangiopancreatography in Indeterminate Biliary Strictures: A Meta-Analysis
}

\author{
Junjie Mi (D614660@163.com ) \\ Shanxi Provincial People's Hospital \\ Xiaofang Han \\ Shanxi Provincial People's Hospital \\ Rong Wang \\ Shanxi Provincial People's Hospital \\ Ruijun Ma \\ Shanxi Provincial People's Hospital \\ Danyu Zhao \\ Shanxi Provincial People's Hospital
}

\section{Research Article}

Keywords: optical biopsy, endoscopic retrograde cholangiopancreatography (ERCP), biliary strictures, microscopic images

Posted Date: November 8th, 2021

DOI: https://doi.org/10.21203/rs.3.rs-1014109/v1

License: @) (i) This work is licensed under a Creative Commons Attribution 4.0 International License. Read Full License 


\section{Abstract}

Probe-based confocal laser endomicroscopy (pCLE), also known as optical biopsy, is a new endoscopic technique that provides real-time magnification of 1000x microscopic tissue information to diagnose indeterminate biliary strictures. Tissue sampling by endoscopic retrograde cholangiopancreatography (ERCP) is routinely performed to evaluate indeterminate biliary strictures. To evaluate the accuracy of pCLE and tissue sampling by ERCP in the diagnosis of indeterminate biliary strictures, 18 articles were included from 2008 to 2021 through Embase, PubMed, Web of Science, and Cochrane library databases. The summary estimates for the pCLE diagnosis of indeterminate biliary strictures were: sensitivity 0.88 (95\% confidence interval (Cl), 0.84-0.91); specificity 0.79 (95\% Cl, 0.74-0.83); and Diagnostic Odds Ratio (DOR) 24.63 (95\% $\mathrm{Cl}, 15.76-38.48)$. The summary estimates for tissue sampling by ERCP diagnosis for indeterminate biliary strictures were: sensitivity 0.54 (95\% $\mathrm{Cl}, 0.49-0.59)$; specificity 0.96 (95\% Cl, 0.94-0.98); and DOR 11.31 (95\% Cl 3.90-32.82). The area under the sROC curve of pCLE diagnosis of indeterminate biliary strictures is 0.90 higher than 0.65 of tissue sampling by ERCP. The PCLE is a better approach than tissue sampling by ERCP for the diagnosis of indeterminate biliary strictures by providing real-time microscopic images of the bile ducts.

\section{Introduction}

Biliary strictures are a common disease of the biliary system. The treatment and prognosis of benign and malignant biliary strictures vary greatly, so it is especially important to distinguish between benign and malignant biliary strictures. Although there are various advanced imaging techniques such as peroral choledochoscopy (POCS) and biopsy techniques such as endoscopic ultrasonography guided fine needle aspiration (EUS-FNA) and endoscopic retrograde cholangiopancreatography (ERCP) biopsy and cytology brush, accurate diagnosis of indeterminate biliary strictures still has challenge ${ }^{1}$. Cholangiocarcinoma is the most common malignant tumor causing malignant strictures of the bile duct, and most tumors grow along the bile duct wall instead of radially to form masses ${ }^{2}$. Therefore, cholangiocarcinoma is usually at an advanced stage when diagnosed, and the survival rate of patients is low. Only $20 \%$ of extrahepatic cholangiocarcinoma can be surgically resected at the time of diagnosis, and the vast majority of patients have a poor prognosis of five-year survival rate of less than $20 \%$. At the same time, as many as $15 \%$ of the suspected hilar cholangiocarcinoma were confirmed to be benign by surgical histopathology ${ }^{2,3}$. Thus, an urgent clinical need exists to improve the diagnostic accuracy of indeterminate biliary strictures so that malignant tumors can be detected as early as possible, improving the prognosis for patients and reducing unnecessary surgeries.

Probe-based confocal laser endomicroscopy (pCLE) is a new diagnostic endoscopy approach that provides real-time microscopic tissue information including blood flow, cytoarchitecture, and fluorescein uptake and leakage. The pCLE enables low-energy laser light to be focused and illuminated through its inner small hole to produce a tissue image with a magnification of 1000 times, which improves the accuracy of targeted biopsy and realizes instant optical biopsy ${ }^{4}$. The PCLE has been used to assess the histopathology of the gastrointestinal tract in vivo $^{5,6}$. The accuracy of pCLE in the diagnosis of gastrointestinal tumors has been demonstrated in previous studies ${ }^{7,8}$. To date, pCLE has been studied in a variety of digestive diseases, including ulcerative colitis, colorectal polyps, pancreatic disease, and Barrett's esophagus ${ }^{9-12}$. In the same situation, $\mathrm{PCLE}$ can also enter the intrahepatic and extrahepatic bile ducts to provide real-time enlarged tissue information for bile duct lesions. The PCLE thus improves the accuracy of diagnosing indeterminate biliary strictures. With the development of pCLE technology and the improvement of the diagnostic classification of indeterminate biliary strictures, a series of studies have been published on the diagnosis of indeterminate biliary strictures in $\mathrm{PCLE}{ }^{13-30}$. The present study will perform a meta-analysis to assess the accuracy of pCLE in diagnosing indeterminate biliary strictures and to compare the accuracy of PCLE and ERCP with brush cytology and intraductal biopsy in diagnosing indeterminate biliary strictures.

\section{Results}

\section{Search results and quality assessment}

Searching using the preset search strategy resulted in 1286 records in Figure 1. After eliminating duplicate records, there were 743 records left. 678 records were excluded immediately after a review of titles and abstracts. After reading the full text of the remaining records, 47 records were further excluded due to various reasons. Three records were added after a manual review of the references of the 15 remaining articles retrieved. Eighteen studies published between 2008 and 2021 were finally included in the meta-analysis. In total, there were 750 lesions reported by 18 studies, 12 prospective and 6 retrospectives in Table 1. The quality of the included studies was assessed using the quality assessment of diagnostic accuracy studies (QUADAS) assessment tool as detailed in Figure 2.

\section{Diagnostic accuracy of pCLE in indeterminate biliary strictures}

Eighteen studies evaluated the accuracy of pCLE diagnosis of indeterminate biliary strictures comprising a total of 750 lesions. Figure 3 shows the sensitivity and specificity of PCLE in the diagnosis of indeterminate biliary strictures. The sensitivity range was $0.75-1.00$, and the specificity range was $0.61-1.00$. The pooled sensitivity and specificity (Fixed effects model) were $0.88(95 \% \mathrm{Cl}, 0.84-0.91)$ and $0.79(95 \% \mathrm{Cl}, 0.74-0.83)$, 
respectively. No significant heterogeneity was found in terms of sensitivity and specificity $\left(I^{2}=0.0 \%, I^{2}=33.2 \%\right)$. An indicator of test accuracy, the Diagnostic Odds Ratio (DOR), combines sensitivity and specificity data into one. DOR equals the value of positive likelihood ratio (PLR) divided by the value of negative likelihood ratio (NLR). A higher value indicates that the test has better diagnostic capabilities. The combined PLR, NLR, and DOR were 3.67 (95\% Cl 3.02-4.45), 0.18 (95\% Cl 0.14-0.24, Figure 4) and 24.63 (95\% Cl 15.76-38.48, Figure 5), respectively, and PLR, NLR, and DOR all had $\mathrm{I}^{2}$ values of $0.0 \%$, which indicated that there is no significant heterogeneity. Figure 5 shows the sROC curve of pCLE in the diagnosis of indeterminate biliary strictures. The area under the SROC curve (AUC) is a method of assessing the diagnostic ability, and it represents the total diagnostic ability. The higher the AUC value, the better the diagnostic ability, and a value of 1.0 indicates that the diagnosis has a near-perfect diagnostic ability. The pCLE diagnosis of indeterminate biliary strictures had a fairly high accuracy rate with an AUC value of 0.90 ( $\mathrm{SE}=0.02$ ). Another way to evaluate the diagnostic performance of the sROC curve is the index $\mathrm{Q}$. The current index $\mathrm{Q}$ value was 0.84 , which also shows that the diagnostic accuracy is high.

\section{Diagnostic accuracy of ERCP with brush cytology and intraductal biopsy in indeterminate biliary strictures}

A total of 16 studies including 784 lesions evaluated the accuracy of tissue sampling by ERCP in the diagnosis of indeterminate biliary strictures, among which intraductal biopsy was 9 articles, brush cytology was 4 articles, brush cytology and intraductal biopsy were 3 articles. Figure 3 shows the sensitivity and specificity of ERCP with brush cytology and intraductal biopsy in the diagnosis of indeterminate biliary strictures. The pooled sensitivity and specificity (random effects model) were 0.54 (95\% Cl, 0.49-0.59) and 0.96 (95\% Cl, 0.94-0.98), respectively. We found significant heterogeneity in terms of sensitivity and specificity $\left(I^{2}=78.9 \%, I^{2}=73.7 \%\right)$. The combined PLR, NLR, and DOR were 5.84 (95\% Cl 2.21-15.43), 0.57 (95\% Cl 0.44-0.74, Figure 4), and 11.31 (95\% Cl 3.90-32.82, Figure 5), respectively, and PLR, NLR, and DOR had $\mathrm{I}^{2}$ values of $78.5 \%, 75.2 \%$, and $67.2 \%$, respectively, which indicated that there is significant heterogeneity. The ERCP with brush cytology and intraductal biopsy diagnosis of indeterminate biliary strictures had a poor accuracy with an AUC value of 0.65 (SE=0.09) in Figure 5 . The accuracy of pCLE in diagnosing indeterminate biliary strictures was better than ERCP with brush cytology and intraductal biopsy, with a significant difference with $95 \%$ confidence regions overlapping.

\section{Subgroup analyses}

We conducted subgroups based on the characteristics of pCLE to diagnose indeterminate biliary strictures. To understand the pCLE diagnosis of indeterminate biliary strictures between different subgroups, we conducted a subgroup analysis and compared whether there are significant statistical differences. The subgroup analysis is shown in Table 2. The subgroups are as follows: diagnostic classification, method, mini probe, country, study design, primary sclerosing cholangitis, follow-up, study center, no. of the lesion. There are two diagnostic classifications for pCLE diagnostic indeterminate biliary strictures, the Paris classification and the Miami classification. Although the sensitivity of the Paris classification in diagnosing indeterminate biliary strictures was lower than that of the Miami classification in the subgroup analysis ( 0.75 versus 0.88), the specificity of the Paris classification was higher than that of the Miami classification ( 0.85 versus 0.77 ). Depending on the mode of entry of pCLE into the bile ducts, there are two subgroups: the cholangioscope subgroup and the catheter subgroup. The cholangioscope subgroup has better sensitivity and specificity for diagnosing indeterminate biliary strictures than the catheter subgroup ( 0.92 versus $0.86,0.93$ versus 0.78 ). Two common Mini probes for diagnosing indeterminate biliary strictures in pCLE are the Gastroflex and CholangioFlex. The sensitivity and specificity of the diagnosis of indeterminate biliary strictures in the Gastroflex subgroup are better than those in the CholangioFlex subgroup ( 0.93 versus $0.87,0.80$ versus 0.78$)$. Primary sclerosing cholangitis is a common cause of indeterminate biliary strictures. The sensitivity of the primary sclerosing cholangitis subgroup to diagnose indeterminate biliary strictures is higher than that of the subgroup without primary sclerosing cholangitis ( 0.88 versus 0.87 ), but the specificity is lower than that of the subgroup without primary sclerosing cholangitis $(0.70$ versus 0.80$)$. The primary sclerosing cholangitis subgroup has a low specificity in the diagnosis of indeterminate biliary strictures, which may be due to severe inflammation of the bile ducts leading to high false positives. $95 \%$ confidence regions have overlap in all different subgroup comparisons, indicating that the differences between subgroups are not statistically significant.

\section{Publication bias and sensitivity analysis}

Deek's funnel plot was used to analyze the potential publication bias of the meta-analysis. Deek's test showed a value of 0.47 (95\% $\mathrm{Cl}-4.95-$ 10.32), and these suggested no possibility of publication bias in Figure 6. There was also no significant threshold effect by Spearman correlation coefficient on pCLE diagnosis of indeterminate biliary strictures (Spearman correlation coefficient: $-0.07 ; p=0.77$ ). For sensitivity analysis, each study was excluded in turn, and the AUC was recalculated for the remaining articles. Yang's study had the greatest impact on the results of the meta-analysis, taking the AUC from 0.9037 to $0.9092(+0.6 \%)^{15}$. The results of the sensitivity analysis showed that the diagnostic efficacy of pCLE for indeterminate biliary strictures remained stable at high levels.

\section{Discussion}

The identification of benign and malignant biliary strictures is critical to patient treatment and prognosis. Early diagnosis of benign biliary strictures can reduce unnecessary surgery and costs, and early detection of bile duct malignancies allows for early surgical intervention to 
improve surgical cure rates and improve patient prognosis. However, due to the particularity of the anatomical structure of the bile duct and the way of tumor growth, the current diagnostic methods still have insufficient sensitivity and accuracy. Therefore, accurate diagnosis of indeterminate biliary strictures is still a major clinical problem.

The diagnosis of biliary strictures is a complex and multiple approach. EUS-FNA is a method for obtaining biopsy tissue from biliary lesions and has $87 \%$ sensitivity and $87 \%$ accuracy for the diagnosis of indeterminate biliary strictures in a prospective single-center trial ${ }^{31}$. However, the shortcoming of this method is that it is limited to intrahepatic biliary strictures or proximal extrahepatic biliary strictures. Single-person operation of POCS allows insertion of the extrahepatic bile duct for direct visualization of the lesion and obtaining a targeted biopsy. In a meta-analysis of single-operator POCS including 335 patients, the sensitivity and specificity of visual representation for diagnosing indeterminate biliary strictures were $90 \%$ and $87 \%$, respectively, whereas the sensitivity and specificity of single-operator POCS biopsy for diagnosing indeterminate biliary strictures were $69 \%$ and $98 \%$, respectively ${ }^{32}$. However, differences in the interobserver agreement were found in various studies, which may be due to the lack of a standard consensus for the diagnosis of indeterminate biliary strictures in POCS ${ }^{33,34}$. In addition, POCS may have difficulty accessing the intrahepatic bile duct and obtaining sufficient tissue samples to confirm a malignancy, but pCLE allows adequate visualization of intrahepatic bile duct strictures because of its small diameter ${ }^{14}$. pCLE has a high accuracy rate in both intrahepatic and extrahepatic biliary strictures due to its fine outer diameter and access to the intrahepatic bile ducts, and there is no statistical difference between intrahepatic (100\%) and extrahepatic biliary strictures $(86 \%)^{17}$. In addition, for indeterminate biliary strictures where the mass is not visible on the image, it is difficult to obtain the specimen by biopsy, but pCLE can obtain a high diagnostic accuracy of $79 \%$ and an negative predictive value (NPV) of $100 \%{ }^{30}$. pCLE is a new imaging technique capable of providing real-time microscopic tissue information in vivo to obtain images of the GI epithelium and sub epithelium at 1,000 magnification during endoscopy. Real-time high-resolution histological diagnosis of mucosa and submucosa tissue structure can achieve the purpose of real-time optical biopsy ${ }^{35}$. As an emerging endoscopic technique, pCLE is gradually spreading worldwide, and the recent American Society for Gastrointestinal Endoscopy guidelines on the management of biliary tract tumors mention pCLE as a useful alternative to existing diagnostic workup ${ }^{36}$. In many studies, pCLE has high sensitivity and accuracy for the diagnosis of indeterminate biliary strictures.

The meta-analysis showed that pCLE as a diagnostic tool for indeterminate biliary strictures had a pooled sensitivity of 0.88 and a pooled specificity of 0.79 . We reported that the index Q value of SROC was 0.84 and AUC was 0.90 , indicating that the overall diagnostic accuracy is very high. The range of DOR is from 0 to infinity, which can be used as an overall assessment of the accuracy of diagnosis. When the DOR value is 1.0 , the test is an invalid diagnostic method ${ }^{37}$. Our study reported a pooled DOR of 24.63, which also illustrates the high overall diagnostic accuracy. Because PLR and NLR are more easily applied in the clinical setting, they were used as an indicator to assess diagnostic accuracy in the current meta-analysis. A PLR value of 3.67 indicated that patients with malignant biliary strictures were approximately three times more likely to be positive compared to patients without malignant biliary strictures. In contrast, the NLR is found to be 0.18 , which means that if the pCLE test is negative, the patient has about a $18 \%$ chance of having malignant biliary strictures. Therefore, based on current data, PLR and NLR may be used as valuable tools for diagnosing uncertain biliary strictures in pCLE in the future.

We noted differences in diagnostic accuracy between subgroups. The diagnosis of pCLE for indeterminate biliary strictures has a Miami classification and a Paris classification, and we compared the diagnostic accuracy of the Miami and Paris classifications in a subgroup analysis. The Miami classification of pCLE diagnostic indeterminate biliary strictures was developed in 2012 and tested by a multicenter study $^{38}$. The image features of Miami classification are shown in Table 3 . In order to improve the specificity of pCLE diagnosis of indeterminate biliary strictures and reduce the false positive rate related to chronic inflammation, Caillol proposed the Paris classification related to benign inflammation changes ${ }^{28}$. The Paris classification is based on the Miami classification with the addition of 4 image features of benign inflammatory stenosis as shown in Table 3. The use of the Paris classification to evaluate the diagnostic accuracy of pCLE for indeterminate biliary strictures shows that the Paris classification can improve the specificity of the diagnosis of indeterminate biliary strictures ${ }^{24,29}$. Similar results were obtained by using the Paris classification in the subgroup of the present study, improving the specificity of the diagnosis from $77.6 \%$ to $85.0 \%$. In future studies, each criterion of the Paris classification scheme for diagnosis of indeterminate biliary strictures needs to be evaluated and appropriate thresholds need to be set. In addition, the thickness of the reticular structure in the Paris classification also needs to be quantified.

Because pCLE can reach the biliary strictures by a catheter or a cholangioscopy, we compared the diagnostic accuracy of the two approaches in a subgroup analysis. The pCLE may reach the biliary strictures more accurately by direct visualization with a cholangioscopy, while the pCLE can only reach the biliary strictures by catheter under fluoroscopic guidance. The cholangioscopy can adjust the angle in the bile duct to make the mini probe to the site of interest diagnose indeterminate biliary strictures in pCLE and obtain a high accuracy of $93.3 \%$. There are similar results in the present study, where the sensitivity and specificity of pCLE for the diagnosis of indeterminate biliary strictures in the cholangioscopy subgroup were higher at $92.3 \%$ and $93.5 \%$, respectively. Additionally, pCLE operators' skills should be improved through training, as highly skilled operators are able to achieve high accuracy ${ }^{16,18,39}$. 
In most pCLE diagnostic studies on indeterminate biliary strictures, the CholangioFlex mini probe was used, which has an outer diameter of less than $1 \mathrm{~mm}$ and a resolution of $3.5 \mathrm{~mm}$. However, some studies have used the GastroFlex mini probe, which increases the number of optical fibers and has better resolution and image quality ${ }^{19,22,23}$. Good image quality achieved $100 \%$ accuracy while poor image accuracy was only $79 \%$, indicating that good image quality can significantly improve accuracy ${ }^{28}$. In addition, the Gastroflex probe had a larger outer diameter but no reduction in cannulation rate ${ }^{19}$. More accurate diagnoses were obtained in some studies using the Gastroflex probe, but the small number of Gastroflex probes used in these studies will require further confirmation in studies with large sample sizes. In the subgroup analysis of this study, the sensitivity and specificity of pCLE in the subgroup of using the GastroFlex mini probe for the diagnosis of indeterminate biliary strictures reached $93.3 \%$ and $80.6 \%$. However, the diagnostic performance of the GastroFlex mini-probe will need to be further clarified by multicenter prospective studies in the future. In addition, if some objective diagnostic methods such as artificial intelligence and signal-to-noise ratio are used, it may improve the sensitivity and specificity of pCLE to diagnose indeterminate biliary strictures ${ }^{20}$.

Brush cytology and intraductal biopsy by ERCP are routinely performed to evaluate indeterminate biliary strictures ${ }^{40}$. We performed a metaanalysis of brush cytology and intraductal biopsies by ERCP. The meta-analysis showed that ERCP with brush cytology and intraductal biopsy as a diagnostic tool for indeterminate biliary strictures has a pooled sensitivity of 0.54 and a good specificity of 0.96 . We reported that the index $\mathrm{Q}$ value of SROC is 0.61 and AUC is 0.65 , indicating that the overall diagnostic accuracy is poor. There is no overlap between the $95 \%$ AUC confidence intervals of pCLE and brush cytology and intraductal biopsy in the diagnosis of indeterminate biliary strictures, indicating that there are significant differences between the two groups. The sensitivity of 0.54 for ERCP with brush cytology and intraductal biopsy is indeed disappointing. The reasons for the low sensitivity could be tumor-associated fibrosis, submucosal spread, or bile duct compression by external lesions. Desmoplastic tumors have a relatively small number of cells and are very firm, making sampling very difficult. Another reason for the poor diagnosis of ERCP with brush cytology and intraductal biopsy is the random sampling of tissues. The pCLE also allows for a more accurate diagnosis by locating different biliary strictures in real time. ERCP with brush cytology and intraductal biopsy combined with pCLE can obtain a more accurate diagnosis ${ }^{18,30}$

This study has several limitations. First, as most of the included studies were retrospective studies, and there was potential selective bias needing further prospective studies to confirm. Secondly, the poor quality of some of the included studies may affect the results of the metaanalysis. Third, some patients in some of the included studies were followed up rather than pathology as the gold standard.

In conclusion, pCLE is a reliable and accurate method for diagnosing indeterminate biliary strictures, especially when reaching the biliary strictures by cholangioscopy. However, pCLE is expensive compared to common ERCP, and future studies are needed to confirm the costeffectiveness. With the development of diagnostic classifications and advances in technology, pCLE will improve the accuracy of diagnosing indeterminate biliary strictures. The pCLE has the potential to overcome the limitations inherent in tissue sampling by ERCP by providing realtime microscopic images of the bile ducts to make an accurate diagnosis of indeterminate biliary strictures.

\section{Materials And Methods}

\section{Search strategy and study selection.}

The study was conducted in accordance with the PRISMA (Preferred Reporting Project for Systematic Reviews and Meta-Analysis) guidelines. The deadline for searching PubMed, Web of Science, Cochrane Library, Embase is October 2021. Search method: $\bigotimes^{\prime \prime p r o b e-b a s e d ~ c o n f o c a l ~ l a s e r ~}$ endomicroscopy" or "confocal laser scanning microscopy" or "confocal microscopy" or "confocal endomicroscopy" or "endomicroscopy" or

“pCLE" or “CLE” $\triangle A N D \otimes " b i l e ~ d u c t$ carcinoma" or "bile duct" or "bile duct neoplasms" or "biliary strictures" or "indeterminate biliary strictures" $₫$. The bibliographies of the retrieved articles were carefully screened for potentially relevant study and the search was not limited to any specific language. Data searching was performed independently by two reviewers (Mi and Han), and disagreements were resolved by consensus.

The studies included in the meta-analysis met the following criteria: (1) The included studies were all published proposing the diagnosis of pCLE on indeterminate biliary strictures; (2) All studies included for the diagnosis of indeterminate biliary strictures had pathology and follow-up as the gold standard for final diagnosis; (3) The number of true positives (TP), false positives (FP), true negatives (TN) and false negatives (FN) can be obtained directly or indirectly in the study. Exclusion criteria: (1) Conference abstracts, letters to editors, reviews, case reports, comments, editorials; (2) pCLE diagnosis of pancreaticobiliary strictures; (3) animal or pediatric studies; (4) pCLE diagnosis of ampullary strictures; (5) artificial intelligence-assisted pCLE.

\section{Data extraction and quality assessment.}

Data extraction for each selected study was performed independently by two reviewers (Mi and Wang), and disagreements were resolved by consensus. The extracted content included the general information of the study, such as year of publication, first author, country, diagnostic classification, mode of entry into the bile duct, follow-up time, study design, number of research centers, type of microprobe, and presence of primary sclerosing cholangitis. To assess the quality and potential bias of the studies, QUADAS was applied to evaluate the quality of the 
included studies. Each eligible study was independently evaluated by two authors (Ma and Zhao), and differences were resolved through discussions with the third author (Mi).

\section{Statistical analyses}

Estimates of diagnostic accuracy for pooled studies, including sensitivity, specificity, PLR, NLR, and DOR were calculated. Pooled results were based on the presence of significant heterogeneity using either the fixed effects model (Mantel-Haenszel method) or the random effects model (DerSimonian-Laird method). In the present meta-analysis, the Cochrane Q test was used to assess heterogeneity between studies, with heterogeneity between studies rather than chance expressed as a percentage $\left(\mathrm{I}^{2}\right)$. More than $50 \%$ of the $\mathrm{I}^{2}$ were considered to be significantly heterogeneous, implying the use of a random effects model rather than a fixed effects model approach to calculate the pooled estimates. The SROC using the method of Moses and colleagues is a comprehensive indicator to evaluate the accuracy of diagnosis. sROC is a graph of the functional relationship between the true positive rate and the false positive rate based on a linear regression model. Overlapping $95 \% \mathrm{Cl}$ for the given values were determined as qualitative methods indicating no significant differences. To explore the accuracy of pCLE diagnostic indeterminate biliary strictures in different subgroups, a subgroup analysis was performed according to the following: year of publication, first author, country, diagnostic classification, mode of entry into the bile duct, follow-up time, study design, number of research centers, type of microprobe, and presence of primary sclerosing cholangitis. The present meta-analysis used Deek's test and funnel plot analysis for publication bias.

Meta-DiSc software version 1.4 was used to test the diagnostic accuracy, including sensitivity, specificity, PLR, NLR, DOR, sROC, and subgroups. The Spearman's correlation coefficient was used to assess the threshold effect using Meta-DiSc software version 1.4. STATA software version 16.0 was used to analyze publication bias. The quality of the included studies was assessed using Review Manager5.3 software. To assess the robustness of the synthesized results, sensitivity analyses will be conducted. The significance level was measured at $\mathrm{P}<0.05$.

\section{Declarations}

Financial disclosure: There are no financial conflicts of interest to disclose.

Funding $\square$ Any funding agency did not involve in this study.

\section{Author contributions:}

(1) Conception and design: Junjie Mi, Xiaofang Han.

(2) Administrative support: Junjie Mi, Xiaofang Han.

(3) Database management and statistical analysis: Ruijun Ma, Rong Wang.

(4) Collection and assembly of data: Junjie Mi, Rong Wang.

(5) Data analysis and interpretation: Xiaofang Han, Danyu Zhao.

(6) Each eligible study was independently evaluated by Ruijun Ma and Danyu Zhao.

(7) Manuscript writing: All authors.

(8) Final approval of manuscript: All authors.

Conflicts of interest: There are no conflicts of interest associated with this study.

\section{References}

1. Victor, D. W., Sherman, S., Karakan, T. \& Khashab, M. A. Current endoscopic approach to indeterminate biliary strictures. World J. Gastroenterol. 18, 6197-6205, doi:10.3748/wjg.v18.i43.6197 (2012).

2. Khan, S. A., Thomas, H. C., Davidson, B. R. \& Taylor-Robinson, S. D. Cholangiocarcinoma. Lancet. 366, 1303-1314, doi:10.1016/S01406736(05)67530-7 (2005).

3. Kambakamba, P. \& DeOliveira, M. L. Perihilar cholangiocarcinoma: paradigms of surgical management. Am. J. Surg. 208, 563-570, doi:10.1016/j.amjsurg.2014.05.017 (2014).

4. Smith, I., Kline, P. E., Gaidhane, M. \& Kahaleh, M. A review on the use of confocal laser endomicroscopy in the bile duct. Gastroenterol Res Pract. 2012, 454717, doi:10.1155/2012/454717 (2012). 
5. Safatle-Ribeiro, A. V. et al. Probe-based confocal endomicroscopy is accurate for differentiating gastric lesions in patients in a Western center. Chin. J. Cancer Res. 30, 546-552, doi:10.21147/j.issn.1000-9604.2018.05.08 (2018).

6. Capuano, A. et al. The Probe Based Confocal Laser Endomicroscopy (pCLE) in Locally Advanced Gastric Cancer: A Powerful Technique for Real-Time Analysis of Vasculature. Front. Oncol. 9, 513, doi:10.3389/fonc.2019.00513 (2019).

7. Kollar, M. et al. Probe-based confocal laser endomicroscopy versus biopsies in the diagnostics of oesophageal and gastric lesions: A prospective, pathologist-blinded study. United European Gastroenterol J. 8, 436-443, doi:10.1177/2050640620904865 (2020).

8. Kim, B. et al. Probe-based confocal laser endomicroscopy for evaluating the submucosal invasion of colorectal neoplasms. Surg Endosc. 31, 594-601, doi:10.1007/s00464-016-5003-x (2017).

9. Rahmi, G. et al. Probe-based Confocal Laser Endomicroscopy for In Vivo Assessment of Histological Healing in Ulcerative Colitis: Development and Validation of the ENHANCE Index. J Crohns Colitis. 15, 994-999, doi:10.1093/ecco-jcc/jjaa255 (2021).

10. Nakaoka, K. et al. Probe-based confocal laser endomicroscopy for the diagnosis of pancreatic ductal structures. J Gastroenterol Hepatol . 36, 118-124, doi:10.1111/jgh.15111 (2021).

11. Caillol, F. et al. Probe confocal laser endomicroscopy in the therapeutic endoscopic management of Barrett's dysplasia. Ann Gastroenterol. 30, 295-301, doi:10.20524/aog.2017.0138 (2017).

12. Belderbos, T. D. G., van Oijen, M. G. H., Moons, L. M. G. \& Siersema, P. D. Implementation of real-time probe-based confocal laser endomicroscopy (pCLE) for differentiation of colorectal polyps during routine colonoscopy. Endosc Int Open. 5, E1104-E1110, doi:10.1055/s-0043-117948 (2017).

13. Solodinina, E. N., Fomicheva, N. V. \& Ulyanov, D. N. Confocal laser endomicroscopy in the diagnosis of extrahepatic bile duct diseases. Annals of HPB Surgery. 24, 11-16, doi:10.16931/1995-5464.2019111-16 (2019).

14. Heif, M., Yen, R. D. \& Shah, R. J. ERCP with probe-based confocal laser endomicroscopy for the evaluation of dominant biliary stenoses in primary sclerosing cholangitis patients. Dig Dis Sci. 58, 2068-2074, doi:10.1007/s10620-013-2608-y (2013).

15. Yang, J. F. et al. Diagnostic accuracy of directed cholangioscopic biopsies and confocal laser endomicroscopy in cytology-negative indeterminate bile duct stricture: a multicenter comparison trial. Minerva Gastroenterol. Dietol. 62, 227-233 (2016).

16. Taunk, P. et al. Improved classification of indeterminate biliary strictures by probe-based confocal laser endomicroscopy using the Paris Criteria following biliary stenting. J Gastroenterol Hepatol. 32, 1778-1783, doi:10.1111/jgh.13782 (2017).

17. Tanisaka, Y. et al. Diagnosis of Biliary Strictures Using Probe-Based Confocal Laser Endomicroscopy under the Direct View of Peroral Cholangioscopy: Results of a Prospective Study (with Video). Gastroenterol Res Pract. 2020, 6342439, doi:10.1155/2020/6342439 (2020).

18. Slivka, A. et al. Validation of the diagnostic accuracy of probe-based confocal laser endomicroscopy for the characterization of indeterminate biliary strictures: results of a prospective multicenter international study. Gastrointest Endosc. 81, 282-290, doi:10.1016/j.gie.2014.10.009 (2015).

19. Shieh, F. K., Drumm, H., Nathanson, M. H. \& Jamidar, P. A. High-definition confocal endomicroscopy of the common bile duct. J. Clin. Gastroenterol. 46, 401-406 (2012).

20. Meining, A. et al. Detection of cholangiocarcinoma in vivo using miniprobe-based confocal fluorescence microscopy. Clin Gastroenterol Hepatol. 6, 1057-1060, doi:10.1016/j.cgh.2008.04.014 (2008).

21. Martínek, J. et al. Confocal laser endomicroscopy in diagnosing indeterminate biliary strictures and pancreatic lesions a prospective pilot study. Rozhl Chir. 99, 258-265, doi:10.33699/pis.2020.99.6.258-265 (2020).

22. Lohr, J. M. et al. Outcome of probe-based confocal laser endomicroscopy (pCLE) during endoscopic retrograde cholangiopancreatography: A single-center prospective study in 45 patients. United European Gastroenterol J . 3, 551-560, doi:10.1177/2050640615579806 (2015).

23. Loeser, C. S., Robert, M. E., Mennone, A., Nathanson, M. H. \& Jamidar, P. Confocal endomicroscopic examination of malignant biliary strictures and histologic correlation with lymphatics. J. Clin. Gastroenterol. 45, 246-252 (2011).

24. Kahaleh, M. et al. Probe-based confocal laser endomicroscopy for indeterminate biliary strictures: refinement of the image interpretation classification. Gastroenterol Res Pract. 2015, 675210, doi:10.1155/2015/675210 (2015).

25. Han, S. et al. Probe-based confocal laser endomicroscopy in the evaluation of dominant strictures in patients with primary sclerosing cholangitis: results of a U.S. multicenter prospective trial. Gastrointest Endosc. 94, 569-576, doi:10.1016/j.gie.2021.03.027 (2021).

26. Giovannini, M. et al. Results of a phase I-Il study on intraductal confocal microscopy (IDCM) in patients with common bile duct (CBD) stenosis. Surg Endosc. 25, 2247-2253, doi:10.1007/s00464-010-1542-8 (2011).

27. Dubow, M., Tatman, P. D. \& Shah, R. J. Individual probe based confocal laser endomicroscopy criteria in the analysis of indeterminate biliary strictures. Scand J Gastroenterol. 53, 1358-1363, doi:10.1080/00365521.2018.1512151 (2018).

28. Caillol, F., Filoche, B., Gaidhane, M. \& Kahaleh, M. Refined probe-based confocal laser endomicroscopy classification for biliary strictures: the Paris Classification. Dig Dis Sci. 58, 1784-1789, doi:10.1007/s10620-012-2533-5 (2013). 
29. Caillol, F. et al. Endomicroscopy in bile duct: Inflammation interferes with pCLE applied in the bile duct: A prospective study of 54 patients. United European Gastroenterol J. 1, 120-127, doi:10.1177/2050640613483462 (2013).

30. Caillol, F. et al. Evaluation of pCLE in the bile duct: final results of EMID study : pCLE: impact in the management of bile duct strictures. Surg Endosc. 29, 2661-2668, doi:10.1007/s00464-014-3986-8 (2015).

31. Onda, S. et al. EUS-guided FNA for biliary disease as first-line modality to obtain histological evidence. Therap. Adv. Gastroenterol. 9, 302312, doi:10.1177/1756283X15625584 (2016).

32. Sun, X. et al. Is single-operator peroral cholangioscopy a useful tool for the diagnosis of indeterminate biliary lesion? A systematic review and meta-analysis. Gastrointestinal endoscopy. 82, 79-87, doi:10.1016/j.gie.2014.12.021 (2015).

33. Sethi, A. et al. Interobserver agreement for evaluation of imaging with single operator choledochoscopy: what are we looking at? Dig Liver Dis. 46, 518-522, doi:10.1016/j.dld.2014.02.004 (2014).

34. Sethi, A. et al. Interobserver agreement for single operator choledochoscopy imaging: can we do better? Diagn Ther Endosc. 2014, 730731, doi:10.1155/2014/730731 (2014).

35. Hurlstone, D. P. et al. In vivo confocal laser scanning chromo-endomicroscopy of colorectal neoplasia: changing the technological paradigm. Histopathology 52, 417-426, doi:10.1111/j.1365-2559.2007.02842.x (2008).

36. American Society for Gastrointestinal Endoscopy Standards of Practice, C. et al. The role of endoscopy in the evaluation and treatment of patients with biliary neoplasia. Gastrointest Endosc. 77, 167-174, doi:10.1016/j.gie.2012.09.029 (2013).

37. Glas, A. S., Lijmer, J. G., Prins, M. H., Bonsel, G. J. \& Bossuyt, P. M. The diagnostic odds ratio: a single indicator of test performance. J. Clin. Epidemiol. 56, 1129-1135, doi:10.1016/s0895-4356(03)00177-x (2003).

38. Meining, A. et al. Direct visualization of indeterminate pancreaticobiliary strictures with probe-based confocal laser endomicroscopy: a multicenter experience. Gastrointest Endosc. 74, 961-968, doi:10.1016/j.gie.2011.05.009 (2011).

39. Talreja, J. P. et al. Pre- and post-training session evaluation for interobserver agreement and diagnostic accuracy of probe-based confocal laser endomicroscopy for biliary strictures. Dig Endosc. 26, 577-580, doi:10.1111/den.12214 (2014).

40. Navaneethan, U. et al. Comparative effectiveness of biliary brush cytology and intraductal biopsy for detection of malignant biliary strictures: a systematic review and meta-analysis. Gastrointest Endosc. 81, 168-176, doi:10.1016/j.gie.2014.09.017 (2015).

\section{Tables}

\section{Table 1. Characteristics of the selected studies.}




\begin{tabular}{|c|c|c|c|c|c|c|c|c|c|}
\hline Author & Year & Country & Method & Design & $\begin{array}{l}\text { Diagnostic } \\
\text { classification }\end{array}$ & $\begin{array}{l}\text { Follow- } \\
\text { up } \\
\text { (month) }\end{array}$ & Center & Mini probe & $\begin{array}{l}\text { Presence } \\
\text { of primary } \\
\text { sclerosing } \\
\text { cholangitis }\end{array}$ \\
\hline Meining & 2008 & Germany & Cholangioscopy & Retrospective & Not used & $>9$ & Single & CholangioFlex & NO \\
\hline Loeser & 2011 & USA & $\begin{array}{l}\text { Catheter or } \\
\text { cholangioscopy }\end{array}$ & Retrospective & Not used & $7-12$ & Single & Gastroflex & NO \\
\hline Giovannini & 2011 & France & Catheter & Retrospective & Not used & Unclear & Single & CholangioFlex & NO \\
\hline Shieh & 2012 & USA & Catheter & Retrospective & Not used & $>12$ & Single & Gastroflex & NO \\
\hline Heif & 2013 & USA & $\begin{array}{l}\text { Catheter or } \\
\text { cholangioscopy }\end{array}$ & Retrospective & Miami & $12-36$ & Single & CholangioFlex & YES \\
\hline Caillol & 2013 & France & $\begin{array}{l}\text { Catheter or } \\
\text { cholangioscopy }\end{array}$ & Retrospective & Miami & 12 & Single & CholangioFlex & NO \\
\hline Caillol & 2013 & France & Catheter & Prospective & Miami & 12 & Single & CholangioFlex & NO \\
\hline Caillol & 2015 & France & Catheter & Retrospective & Miami & 12 & Single & CholangioFlex & NO \\
\hline Kahaleh & 2015 & USA & Catheter & Retrospective & Paris & Unclear & Single & CholangioFlex & NO \\
\hline Slivka & 2015 & USA & Catheter & Prospective & $\begin{array}{l}\text { Miami and } \\
\text { Paris }\end{array}$ & $6-12$ & Multiple & CholangioFlex & NO \\
\hline Löhr & 2015 & Sweden & $\begin{array}{l}\text { Catheter or } \\
\text { cholangioscopy }\end{array}$ & Prospective & $\begin{array}{l}\text { Miami and } \\
\text { Paris }\end{array}$ & $>12$ & Single & Gastroflex & NO \\
\hline Yang & 2016 & USA & $\begin{array}{l}\text { Catheter or } \\
\text { cholangioscopy }\end{array}$ & Retrospective & $\begin{array}{l}\text { Miami and } \\
\text { Paris }\end{array}$ & $>12$ & Multiple & CholangioFlex & NO \\
\hline Taunk & 2017 & USA & $\begin{array}{l}\text { Catheter or } \\
\text { cholangioscopy }\end{array}$ & Retrospective & $\begin{array}{l}\text { Miami and } \\
\text { Paris }\end{array}$ & 12 & Single & CholangioFlex & NO \\
\hline Dubow & 2018 & USA & Unclear & Retrospective & $\begin{array}{l}\text { Miami and } \\
\text { Paris }\end{array}$ & $>6$ & Single & CholangioFlex & NO \\
\hline Solodinina & 2019 & Russia & Cholangioscopy & Retrospective & Unclear & $12-48$ & Single & CholangioFlex & NO \\
\hline Tanisaka & 2020 & Japan & Cholangioscopy & Prospective & Miami & $>12$ & Single & CholangioFlex & NO \\
\hline Martínek & 2020 & Czech & Cholangioscopy & Prospective & $\begin{array}{l}\text { Miami and } \\
\text { Paris }\end{array}$ & 10 & Multiple & CholangioFlex & NO \\
\hline Han & 2021 & USA & $\begin{array}{l}\text { Catheter or } \\
\text { cholangioscopy }\end{array}$ & Prospective & $\begin{array}{l}\text { Miami and } \\
\text { Paris }\end{array}$ & $>12$ & Multiple & CholangioFlex & YES \\
\hline
\end{tabular}

Table 2. Subgroup analysis of diagnostic indices (with $95 \%$ confidence interval) 


\begin{tabular}{|c|c|c|c|c|c|c|}
\hline Subgroup & $\begin{array}{l}\text { No. of } \\
\text { studies }\end{array}$ & $\begin{array}{l}\text { Sensitivity pooled } \\
\varangle \% \bigotimes\end{array}$ & $\begin{array}{l}\text { Specificity pooled } \\
\varangle \% \square\end{array}$ & PLR pooled & NLR pooled & DOR pooled \\
\hline \multicolumn{7}{|l|}{$\begin{array}{l}\text { Diagnostic } \\
\text { classification }\end{array}$} \\
\hline Miami classification & 6 & $88.2 \rrbracket 81.6-93.1 \rrbracket$ & 77.6(68.5-85.1) & $4.27(2.83-6.46)$ & $\begin{array}{l}0.15(0.09- \\
0.25)\end{array}$ & $29.45(13.56-63.95)$ \\
\hline Paris classification & 2 & $75.0(53.3-90.2)$ & $85.0(70.2-94.3)$ & $\begin{array}{l}4.91(2.27- \\
10.63)\end{array}$ & $\begin{array}{l}0.30(0.15- \\
0.60)\end{array}$ & $17.12(4.93-59.48)$ \\
\hline \multicolumn{7}{|l|}{ Method } \\
\hline Cholangioscope & 4 & $92.3(79.1-98.4)$ & $93.5(82,1-98.6)$ & $\begin{array}{l}11.99(4.31- \\
33.35)\end{array}$ & $\begin{array}{l}0.10(0.04- \\
0.27)\end{array}$ & $\begin{array}{l}112.27(24.41- \\
516.43)\end{array}$ \\
\hline Catheter & 6 & $86.4(80.9-90.9)$ & $78.0(69.0-85.4)$ & $3.57(2.51-5.08)$ & $\begin{array}{l}0.19(0.13- \\
0.27)\end{array}$ & $20.24(10.99-37.27)$ \\
\hline \multicolumn{7}{|l|}{ Mini probe } \\
\hline Gastroflex & 3 & 93.3(68.1-99.8) & $80.6(62.5-92.5)$ & $3.97(1.91-8.24)$ & $\begin{array}{l}0.16(0.05- \\
0.58)\end{array}$ & $25.06(4.11-152.74)$ \\
\hline CholangioFlex & 15 & 87.4(83.3-90.8) & $78.6(74.2-82.6)$ & $4.13(3.31-5.16)$ & $\begin{array}{l}0.17(0.12- \\
0.23)\end{array}$ & $25.52(16.49-39.48)$ \\
\hline \multicolumn{7}{|l|}{ Country } \\
\hline Europe & 8 & $88.1(82.2-92.6)$ & 79.7(71.3-86.5) & $4.28(2.95-6.19)$ & $\begin{array}{l}0.14(0.08- \\
0.22)\end{array}$ & $31.11(15.37-62.99)$ \\
\hline USA & 9 & $86.5(80.0-91.4)$ & $77.8(72.4-82.5)$ & $7.78(2.93-4.88)$ & $\begin{array}{l}0.20(0.13- \\
0.29)\end{array}$ & $20.64(11.96-35.63)$ \\
\hline \multicolumn{7}{|l|}{ Study design } \\
\hline Prospective & 6 & $88.5(82.2-93.2)$ & $75.2(67.3-82.0)$ & $3.63(2.58-5.10)$ & $\begin{array}{l}0.15(0.09- \\
0.25)\end{array}$ & $22.70(11.59-44.47)$ \\
\hline Retrospective & 12 & $87.0(81.4-91.4)$ & $80.8(75.5-85.3)$ & $4.50(3.42-5.93)$ & $\begin{array}{l}0.17(0.12- \\
0.25)\end{array}$ & $27.45(15.89-47.44)$ \\
\hline \multicolumn{7}{|l|}{$\begin{array}{l}\text { Presence of primary } \\
\text { sclerosing } \\
\text { cholangitis }\end{array}$} \\
\hline YES & 2 & $88.9(51.8-99.7)$ & $70.0(57.9-80.4)$ & $2.78(1.79-4.30)$ & $\begin{array}{l}0.22(0.05- \\
0.96)\end{array}$ & $12.68(2.14-75.22)$ \\
\hline NO & 16 & $87.6(83.6-91.0)$ & $80.6(76.0-84.7)$ & $4,24(3.38-5.32)$ & $\begin{array}{l}0.16(0.12- \\
0.22)\end{array}$ & $26.88(17.41-41.50)$ \\
\hline \multicolumn{7}{|l|}{ Follow-up (month) } \\
\hline > 12 Months & 11 & 87.8(82.2-92.2) & 78.8(73.1-83.7) & $4.37(3.24-5.90)$ & $\begin{array}{l}0.16(0.11- \\
0.25)\end{array}$ & $27.16(14.96-49.31)$ \\
\hline$<12$ Months & 7 & $87.4(81.2-92.1)$ & 78.8(71.8-84.8) & $3.87(2.85-5.24)$ & $\begin{array}{l}0.17(0.11- \\
0.26)\end{array}$ & $23.73(12.97-43.41)$ \\
\hline \multicolumn{7}{|l|}{ Study center } \\
\hline Single & 14 & $88.0(83.2-91.8)$ & 79.2(73.8-83.9) & $4.36(3.34-5.69)$ & $\begin{array}{l}0.15(0.11- \\
0.22)\end{array}$ & $28.21(16.65-47.80)$ \\
\hline Multiple & 4 & $86.9(78.6-92.8)$ & 78.1(70.5-84.5) & $3.67(2.57-5.23)$ & $\begin{array}{l}0.19(0.11- \\
0.32)\end{array}$ & $20.69(10.08-42.46)$ \\
\hline \multicolumn{7}{|l|}{ No. of lesion } \\
\hline$>50$ & 7 & $87.9(83.0-91.8)$ & 76.8(71.2-81.7) & $3.70(2.88-4.75)$ & $\begin{array}{l}0.16(0.11- \\
0.24)\end{array}$ & $24.30(14.36-41.12)$ \\
\hline$<50$ & 11 & 87.2(79.4-92.8) & $82.5(75.3-88.4)$ & $5.22(3.47-7.85)$ & $\begin{array}{l}0.17(0.11- \\
0.28)\end{array}$ & $27.74(13.50-57.00)$ \\
\hline
\end{tabular}


Table 3. Both the Miami-criteria and Paris-criteria for the diagnosis of indeterminate biliary strictures in pCLE

\begin{tabular}{|lll|}
\hline Tumorous bile duct & Normal bile duct & Inflammatory bile duct \\
\hline Thick white bands $(>20 \mu \mathrm{m})$ & Reticular network of thin dark branching bands $(<20 \mu \mathrm{m})$ & Vascular congestion \\
Thick dark bands $(>40 \mu \mathrm{m})$ & Vessels $(<20 \mu \mathrm{m})$ & Dark granular patterns with scales \\
Dark clumps & Light gray background & Increased inter-glandular space \\
Epithelium & & Thickened reticular structure
\end{tabular}

\section{Figures}

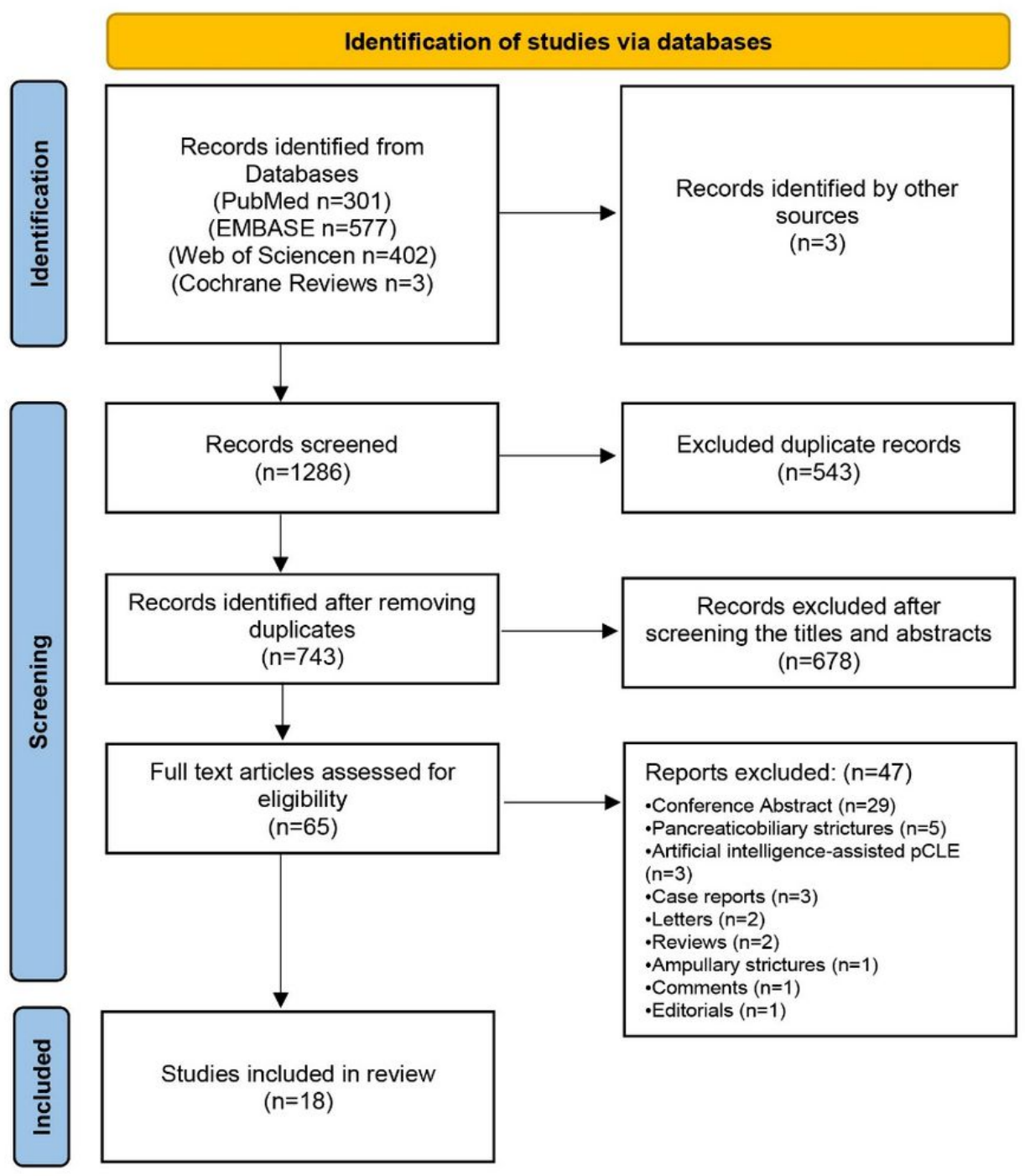

Figure 1

PRISMA flow chart. 


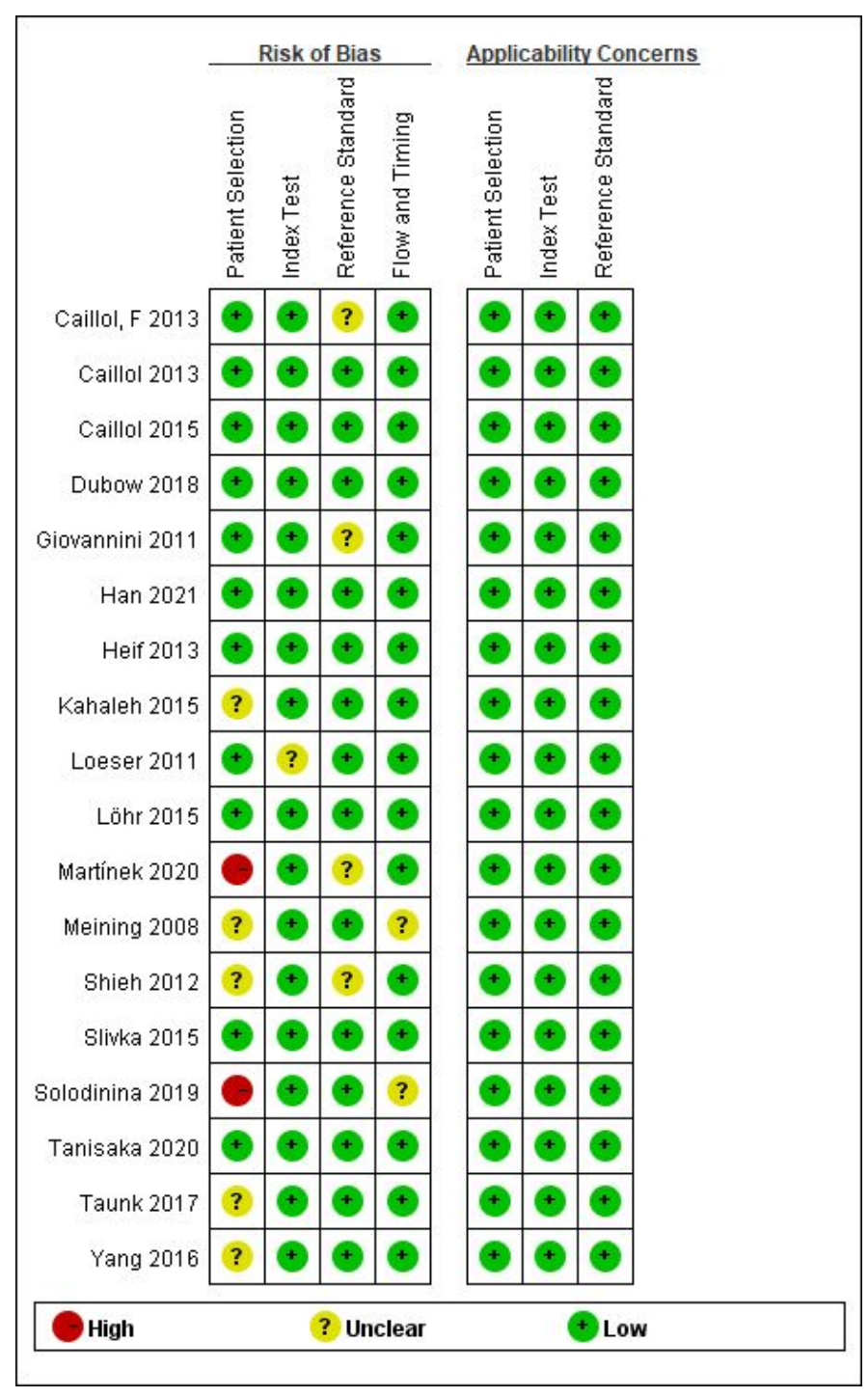

Figure 2

The quality of included studies and the risk of bias. 
(a)

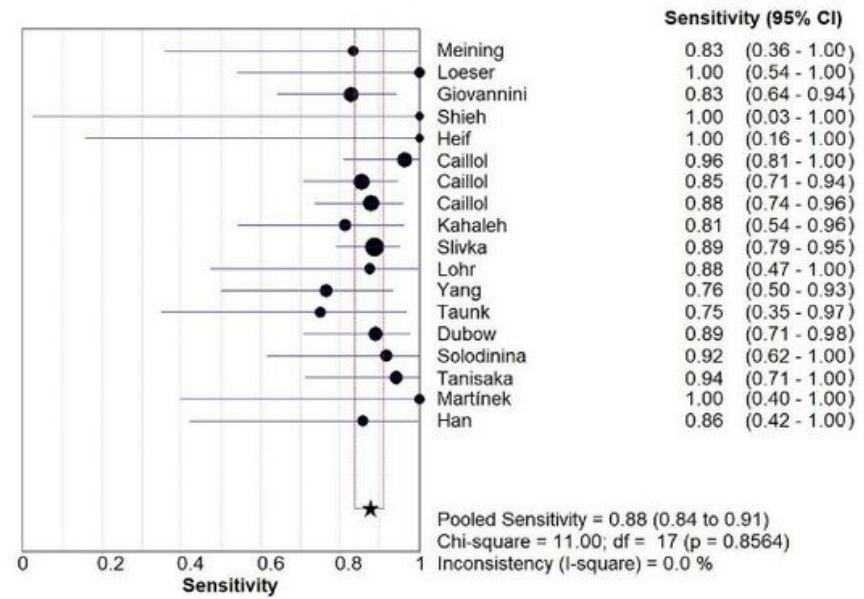

(c)

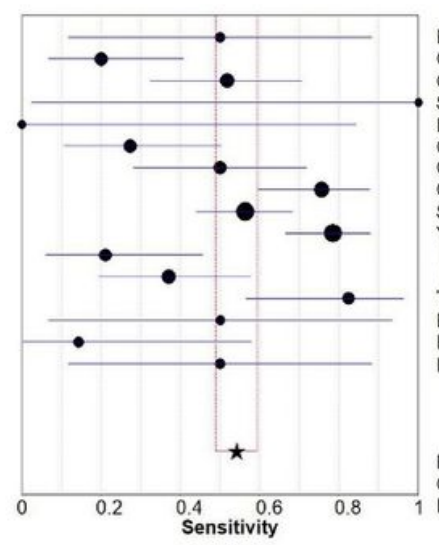
Giovannini Giovannini Shieh Heif

Caillor

Caillol

Slivka

Dubow

Dubow

Tanisaka

Han

Pooled Sensitivity $=0.54(0.49$ to 0.59$)$ Chi-square $=71.18 ; \mathrm{df}=15(\mathrm{p}=0.0000)$ Inconsistency (I-square) $=78.9 \%$

Sensitivity $(95 \% \mathrm{Cl})$

$\begin{array}{ll}0.50 & (0.12-0.88) \\ 0.20 & (0.07-0.41)\end{array}$

$0.52 \quad(0.33-0.71)$

$1.00 \quad(0.03-1.00)$

$0.00 \quad(0.00-0.84)$

$0.27 \quad(0.11-0.50)$

$0.50 \quad(0.28-0.72$

$0.76 \quad(0.60-0.88)$

$0.56 \quad(0.44-0.68)$

$0.78 \quad(0.67-0.88)$

$0.21 \quad(0.06-0.46)$

$0.82 \quad(0.57-0.96)$

$0.50 \quad(0.07-0.93)$

$0.14 \quad(0.00-0.58$

$0.50 \quad(0.12-0.88$ (b)

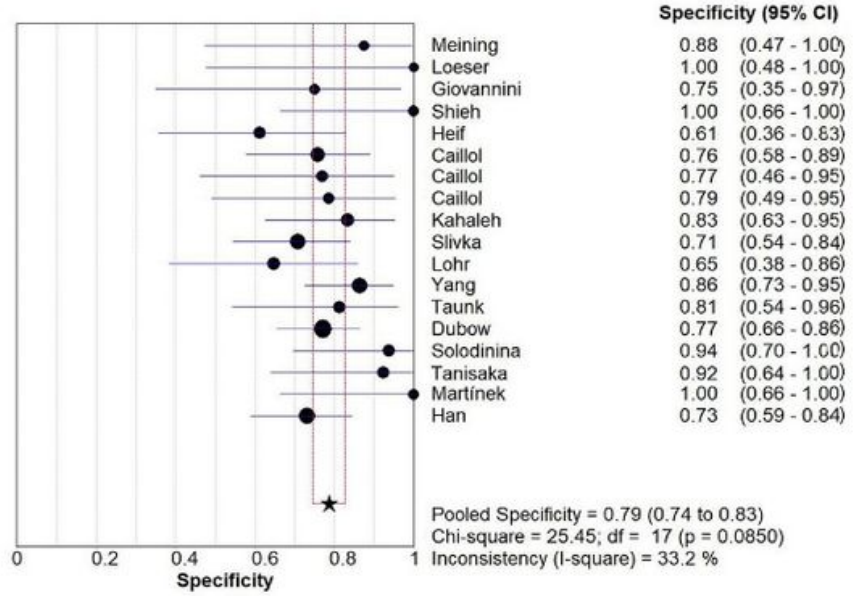

(d)

$0.37 \quad(0.19-0.58$

)


(a)

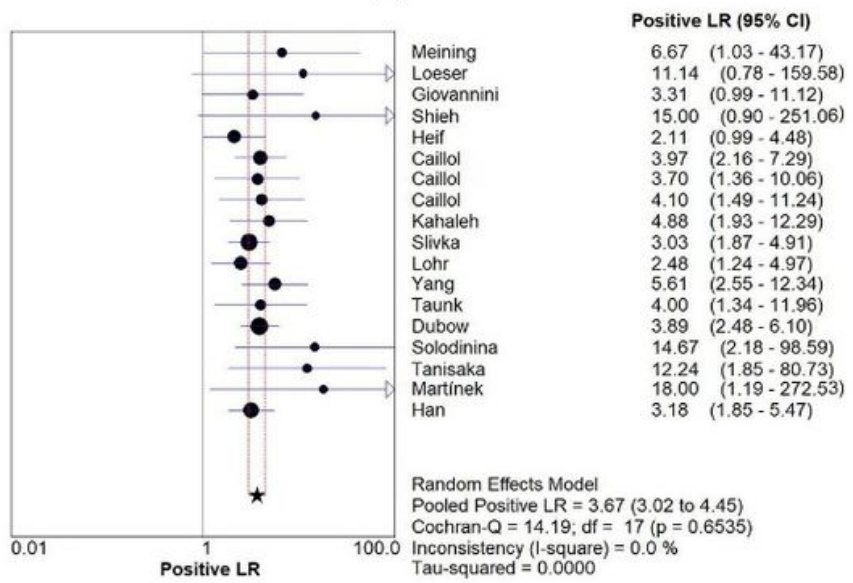

(c)

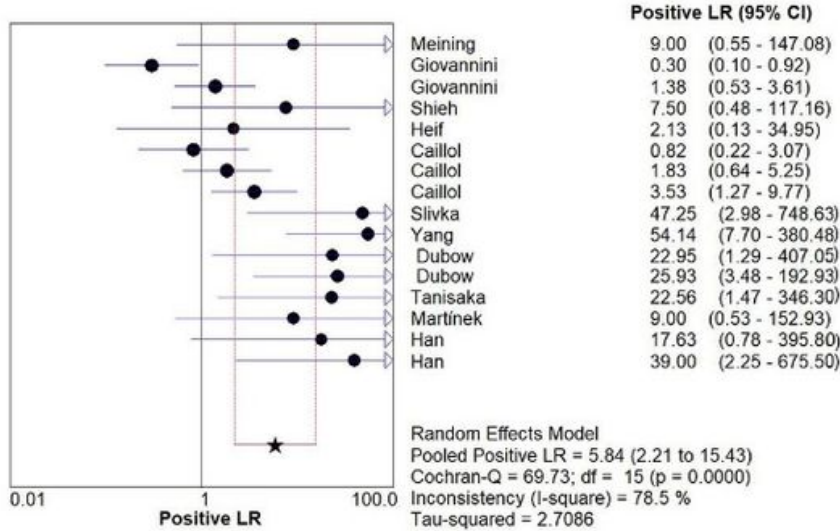

(b)

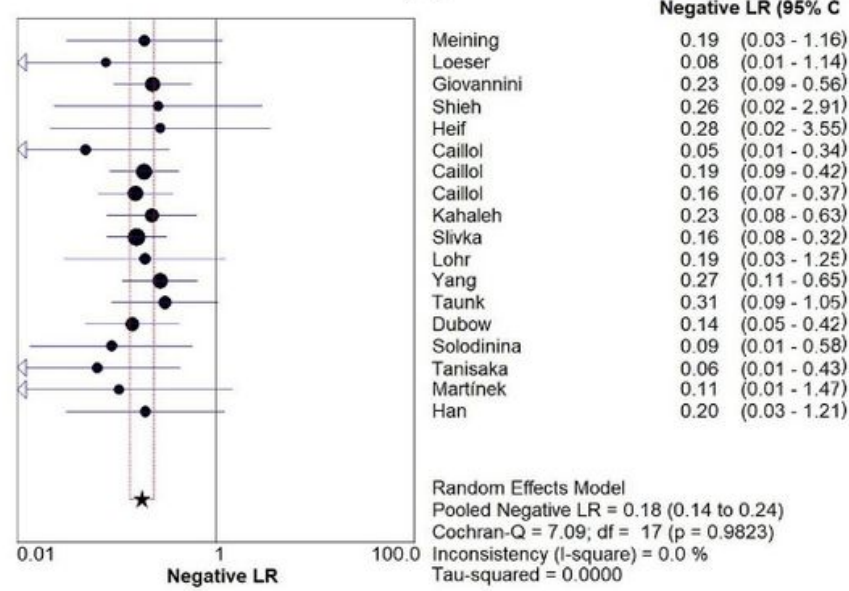

(d)

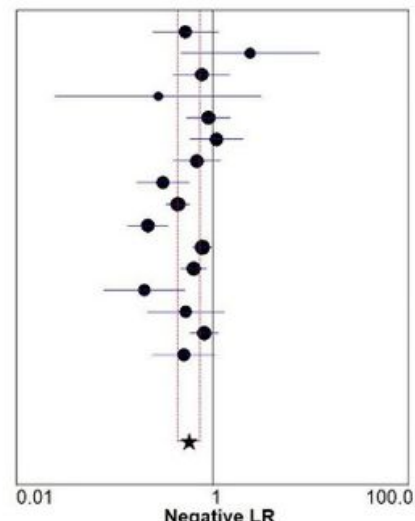

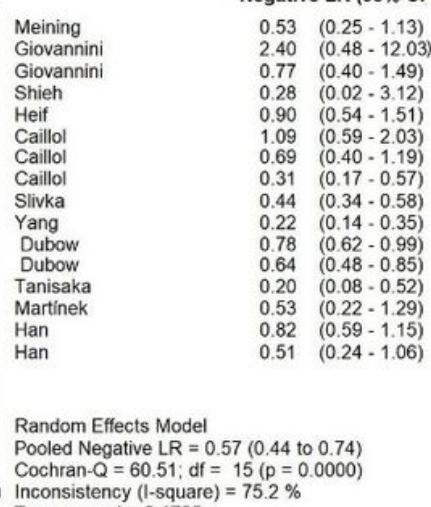

( $(1-$ square $)=75.2 \%$

\section{Figure 4}

Forest plot of PLR and NLR of pCLE $(a, b)$ and tissue sampling by $\operatorname{ERCP}(c, d)$ in diagnosing indeterminate biliary strictures. 
(a)
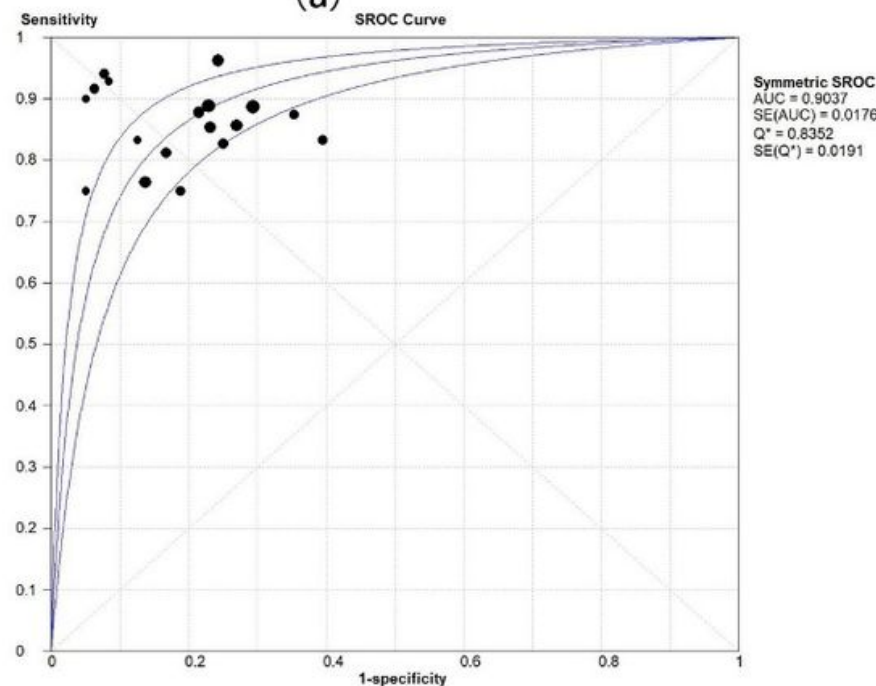

(c)

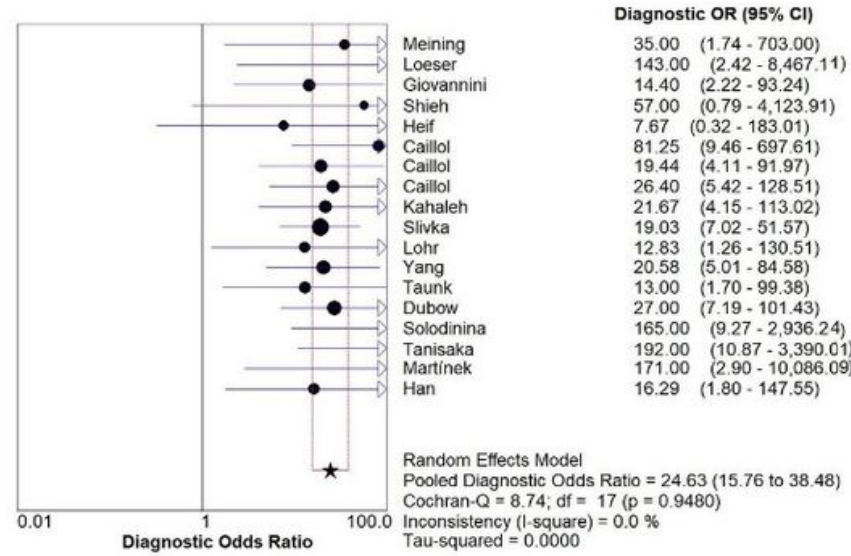

(b)

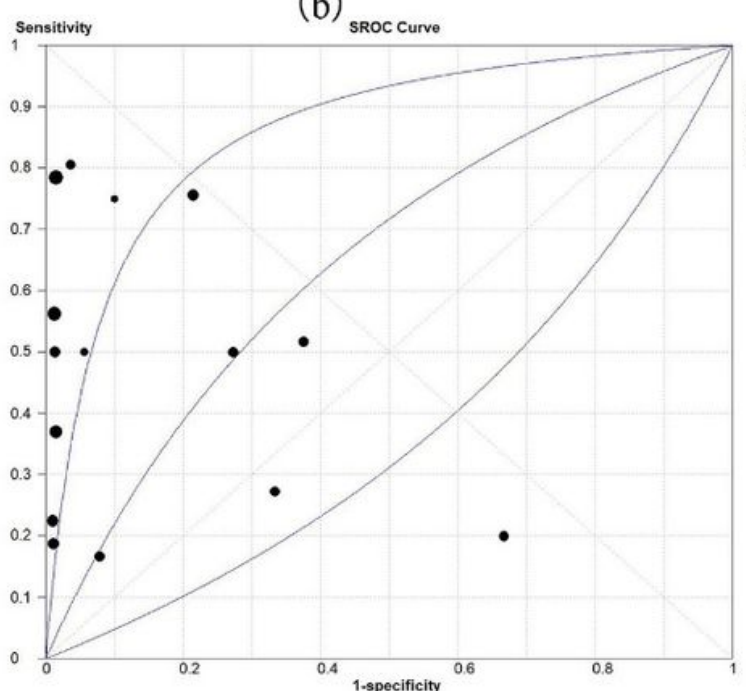

Symmetric SROC $S E(A \cup C)=0.1228$ $Q^{*}=0.6141$
$\operatorname{SE}\left(Q^{*}\right)=0.0951$

(d)

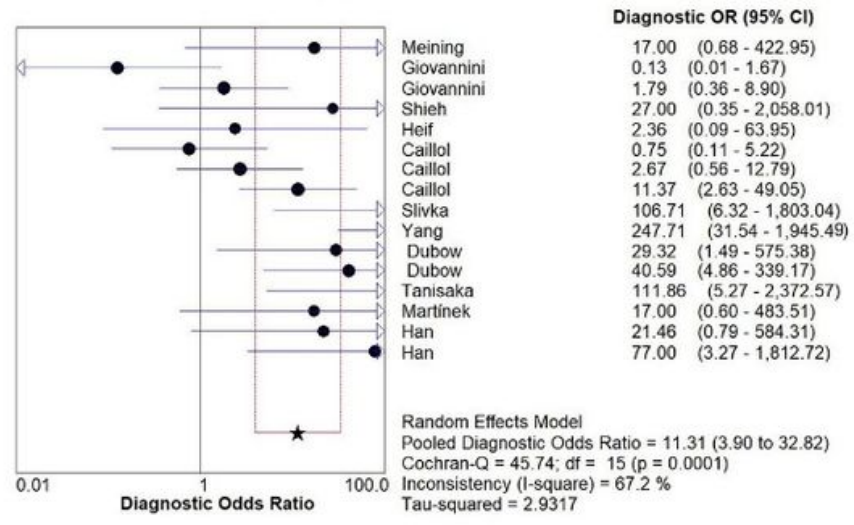

Figure 5

SROC plot and DOR of pCLE $(a, c)$ and tissue sampling by ERCP $(b, d)$ in diagnosing indeterminate biliary strictures. 


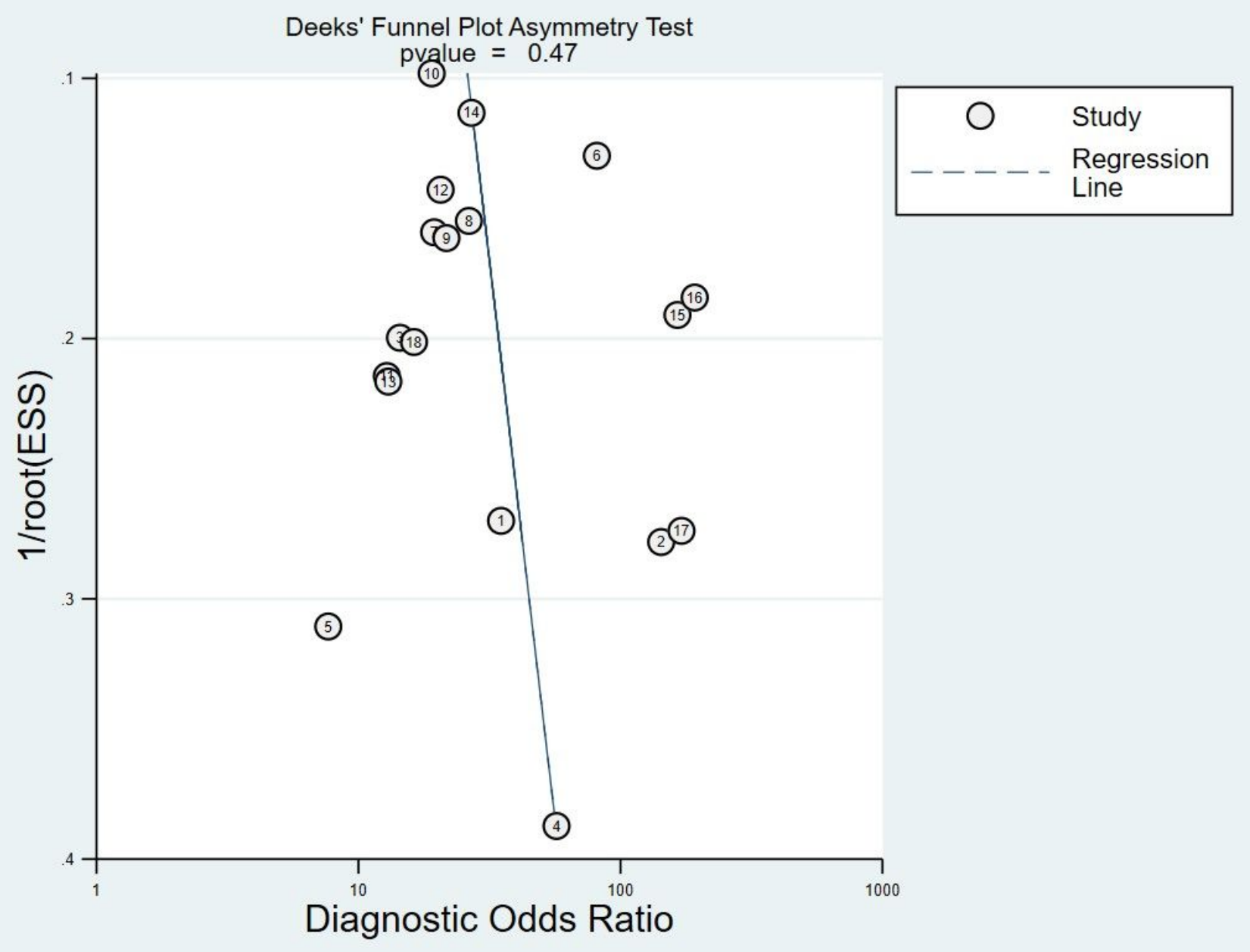

Figure 6

Funnel plot for the evaluation of potential publication bias of selected studies.

\section{Supplementary Files}

This is a list of supplementary files associated with this preprint. Click to download.

- PRISMA2020checklist.docx 